Ann. Zootech., I978, 27 (I), IoI-Io6.

\title{
Note
}

\section{Comportement alimentaire du lapin de garenne élevé en captivité. II. - Modifications induites
par un décalage des périodes d'éclairement}

M. PRUD'HON, J. GOUSSOPOULOS et Y. REYNE:

Station de Physiologie animale, I.N.R.A., E.N.S.A., Place Viala. 34060 Montpellier Cedex (France)

\section{Résumé}

Les caractéristiques du comportement alimentaire de trois lapins de garenne élevés en captivité ont été enregistrées pendant quatre semaines avec un éclairement de 7 heures à 2r heures puis pendant sept semaines avec un éclairement de zo heures à ro heures.

La modification d'horaire lumineux ne modifie pas les caractéristiques quantitatives de la consommation; par contre, la répartition de la consommation au cours du nycthémère change progressivement pour s'adapter au nouvel horaire en quatre semaines environ. Chez certains sujets, cette adaptation n'est pas entièrement achevée au bout de sept semaines.

\section{Introduction}

Dans de nombreuses espèces le rythme d'ingestion d'aliments solides et d'eau est lié plus ou moins étroitement à l'alternance des périodes de lumière et d'obscu. rité, l'utilisation d'un éclairage programmé à heure fixe jouant le rôle de synchroniseur des séquences de comportement alimentaire. Ainsi des espèces comme le Rat (LE MAGNEN et TAllon, 1966), le Chien (Ardisson et al., I974), le Lapin domestique (PRUD'HON et al., I972, SANDERSON et VAN DER WEELF, I975) et plus encore le Lapin de garenne (PRUD'HON et Goussopoulos, I976) ont une 
consommation nocturne prédominante dépendant du programme lumineux appliqué.

Chez le Lapin domestique nous avons montré que l'introduction d'un autre synchroniseur, tel que la distribution d'eau à heure fixe, modifie profondément le comportement alimentaire mais n'abolit pas le rythme de consommation lié à l'alternance lumière-obscurité (PRUD'HON et al., 1975).

En sens inverse on peut penser qu'une modification du programme d'éclairement va entraîner des variations de comportement alimentaire, d'autant plus importantes que celui-ci lui est lié plus étroitement.

Dans cette étude nous analysons les modifications de comportement alimentaire du Lapin de garenne induites par un simple décalage de la période d'éclairement, sans modification de la durée des phases claires et obscures.

\section{Matériel et méthodes}

Trois lapins de garenne, deux femelles et un mâle, recueillis au nid, élevés en captivité en cages grillagées jusqu'à l'âge de 9 mois, ont été placés dans des cages équipées d'un dispositif d'enregistrement graphique des consommations dans les conditions que nous avons décrites précédemment (PRUD'HON et GoussoPOULOS, I976).

Pendant quatre semaines, les lapins de garenne étaient éclairés de 7 à 2I heures, à l'abri complet de la lumière extérieure, soit un rapport lumière/obscurité de I4/Io. A partir du $29^{\text {e }}$ jour nous avons établi une nouvelle répartition des périodes d'obscurité (de Io heures à 20 heures) et d'éclairement (20 heures à Io heures) sans modifier l'importance respective des deux phases (rapport lumière / obscurité de I4/Io), ce qui revient à supprimer en une seule fois II heures de lumière et à ramener la période d'obscurité à un moment où les lapins n'étaient pas habitués à manger.

L'enregistrement des séquences de comportement alimentaire a été poursuivi pendant 7 semaines sous ce nouvel horaire.

Tout au long de l'essai nous avons mesuré chaque jour pour chaque animal la fréquence des repas solides et liquides, les quantités ingérées et leur répartition au cours du nycthémère.

\section{Résultats}

Le décalage de la période de distribution de la lumière a entraîné chez les trois lapins étudiés des modifications analogues de comportement alimentaire qui se sont répercutées sur une période de 4 à 5 semaines selon les individus. Ces modifications sont illustrées sur la figure I où nous avons représenté, pour un lapin, l'évolution jour après jour, des horaires de consommation solide (A) et liquide (B). On notera qu'au cours de la première partie des mesures (lumière de 7 à 2 I heures) le Lapin s'arrête de boire et de manger respectivement $z$ heures et I heure environ avant l'allumage du local, mais il commence aussi à consommer une heure avant la fin de la phase claire.

Après modification de l'horaire d'éclairage le Lapin se met presque immédiate- 
ment à réaliser des prises d'aliment au cours de la nouvelle période obscure tout en continuant à manger à son ancien horaire mais en décalant chaque jour celui-ci. Il y a un véritable glissement qui ne prendra fin que lorsque la consommation sera redevenue nocturne.

Au bout de 4 semaines, le rythme des consommations est redevenu très proche du rythme initial; toutefois, chez les trois sujets étudiés, on note une certaine

SOLIDE SOLID

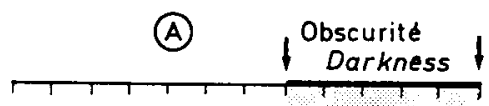

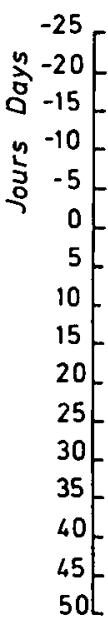

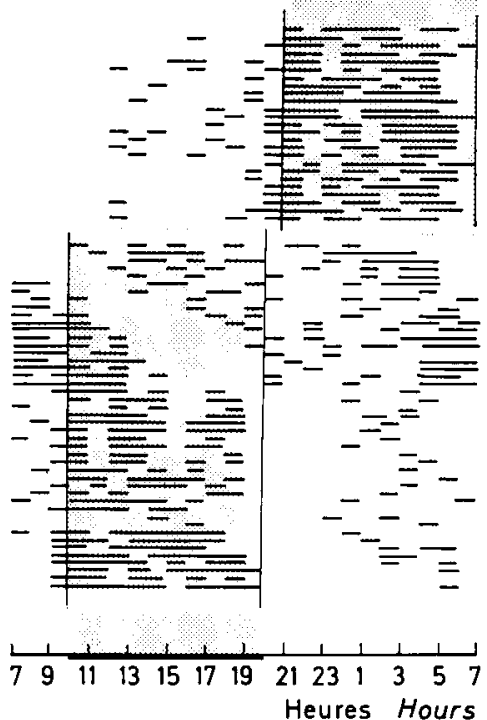

LIOUIDE LIQUID
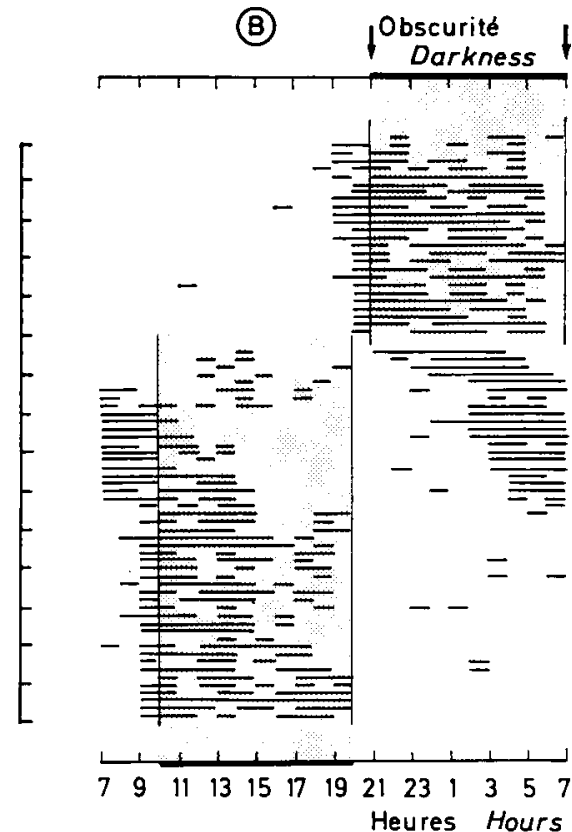

FIG. I. - Horaires des consommations solides $(A)$ et liquides $(B)$ d'un lapin éclairé de 7 heures $\grave{a} 21$ heures puis de 20 heures à Io heures.

- Chaqua ligne représente une journée subdivisée en 24 heures. Si au cours d'une heure le lapin mange ou boit le trait est inserit sur l'espace correspondant à cette heure, si aucun repas n'est pris aucun trait n'est inserit.

Solid $(A)$ and liquid $(B)$ intake pattern of a rabbit on light period from $7:$ oo am to $9: 00$ pm and afterwards from 8:00 pm to IO: 00 am

- Each line represents a day period subdivised in 24 hours day period. If during one hour the rabbit eats or drinks it is recorded in the corresponding period (ink lines).

tendance à maintenir une consommation diurne solide et, chez un sujet, liquide à une heure qui correspond à l'ancienne période d'obscurité (fig. 2).

La durée des mesures ( 7 semaines) n'a pas été suffisante pour que disparaissent ces consommations diurnes, notamment en ce qui concerne les aliments solides.

Sur le plan quantitatif, les perturbations apportées au rythme des consom. mations n'cnt eu aucun effet significatif sur le niveau moyen d'ingestion des aliments solides et liquides (tabl. I).

I'étude plus précise des fréquences et quantités journalières d'aliment con- 

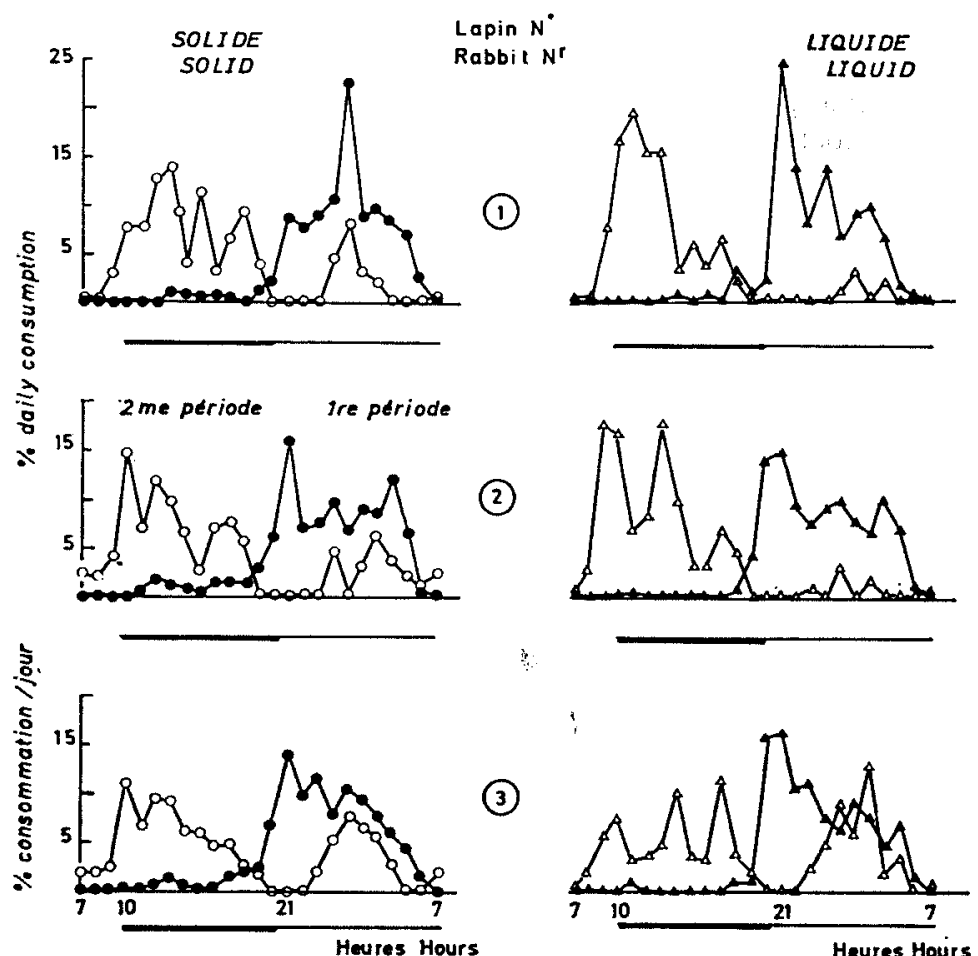

(2)
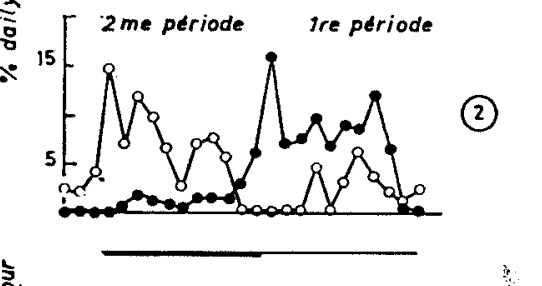

(3)

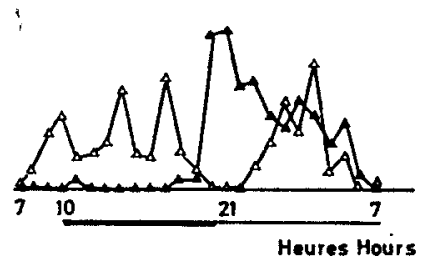

Fig. 2. - Répartition au cours du nycthémère des consommations solides $(A)$ et liquides $(B)$ de trois lapins éclairés d'abord de 9 heures à 21 heures puis de 20 heures à Io heures.

- Ces répartitions sont calculées à partir des consommations réalisées au cours de la dernière semaine d'éclairage 7-2r heures et de la cinquième semaine d'éclairage 20-to heures, c'est-àdire après adaptation au nouvel horaire. Les consommations horaires sont exprimées en pourcentage de la consommation journalière.

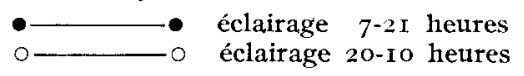

Circadian distribution of feed $(A)$ and water $(B)$ intake of three rabbits on light period from $7: 00$ am to $9: 00 \mathrm{pm}$ and afterwards from $8:$ oo pm to $\mathrm{xo}: \mathrm{oo}$ am.

- The curves are established from amount of feed ingested during the last week of the 7: oo am to 9:00 $\mathrm{pm}$ period and during the 5 th week of the $8: 00$ pm to 10 ; oo am period, i.e. after adaptation to light phase shift.

sommé pendant les 4 semaines qui précèdent et les 7 semaines qui suivent la modification d'horaire d'éclairement ne nous a pas permis de mettre en évidence un effet à court terme de celui-ci.

\section{Discussion et conclusion}

Chez le Lapin de garenne comme chez le Lapin domestique (CHERUBIN, I974) ou le Chien (ARDisson et al., 1974) une modification de l'horaire de distribution de la lumière entraîne une adaptation progressive aux nouvelles conditions d'éclai- 
TABLEAU I

Caractéristiques des consommations au cours des trois semaines précédant ou suivant la modification d'horaive d'éclaivement

Feed intake pattern during the three-weeks preceding

or following artificial day length modification

\begin{tabular}{|c|c|c|c|c|c|}
\hline \multirow[b]{2}{*}{$\begin{array}{c}\text { Lapin } \mathrm{n}^{0} \\
\left(\text { Rabbit } n^{\circ}\right)\end{array}$} & \multirow[b]{2}{*}{$\begin{array}{c}\text { Périodes } \\
(\text { Periods (1)) }\end{array}$} & \multicolumn{2}{|c|}{$\begin{array}{l}\text { Consommation solide } \\
\text { (Daily feed intake) }\end{array}$} & \multicolumn{2}{|c|}{$\begin{array}{l}\text { Consommation liquide } \\
\text { (Daily water intake) }\end{array}$} \\
\hline & & $\begin{array}{c}\text { Fréquence } \\
\text { des prises } \\
\text { (Meal frequency) }\end{array}$ & $\begin{array}{l}\text { Quantité }(g) \\
\text { (Feed intake } \\
\text { level }(g))\end{array}$ & $\begin{array}{l}\text { Fréquence } \\
\text { des prises } \\
\text { (Drinking } \\
\text { frequency) }\end{array}$ & $\begin{array}{c}\text { Quantité }(\mathrm{g}) \\
\text { (Water intake } \\
\text { level }(g))\end{array}$ \\
\hline I. . . & $\left\{\begin{array}{lll}\mathbf{I} \cdot & \cdot \\
\mathbf{2} \cdot & \cdot & \cdot \\
\end{array}\right.$ & $\begin{array}{l}\text { I } 3,8+3,8 \\
\text { г } 0,3+3,3\end{array}$ & $\begin{array}{l}49,5 \div 11,9 \\
41,4 \pm 10,3\end{array}$ & $\begin{array}{l}8,3 \pm 2,3 \\
7,6 \pm 1,8\end{array}$ & $\begin{array}{l}60,4 \pm 13,8 \\
59,2 \doteq 12,0\end{array}$ \\
\hline 2 & $\begin{array}{lll}\text { I. } \cdot & \cdot & \cdot \\
2 . & \cdot & \cdot \\
\end{array}$ & $\begin{array}{r}\text { IO,0 } \pm \text { J,6 } \\
9,4=2,8\end{array}$ & $\begin{array}{lll}37,6 & 6,5 \\
41,6 & 9,6\end{array}$ & $\begin{array}{r}9,6 \pm 2,5 \\
10,3 \pm 2,9 \\
\end{array}$ & $\begin{array}{l}57,4 \pm \text { r }, 8 \\
64, \mathrm{I} \pm \mathrm{r} 3,8 \\
\end{array}$ \\
\hline $3 \cdot \cdot \cdot$ & $\left\{\begin{array}{llll}I \cdot \cdot & \cdot & \cdot & \cdot \\
2 \cdot & \cdot & \cdot & \cdot\end{array}\right.$ & $\begin{array}{l}I 8, I \pm 4,6 \\
16,3 \pm 6,0\end{array}$ & $\begin{array}{lll}37,5 & 6,5 \\
35,0 & \cdots & 7,6\end{array}$ & $\begin{array}{l}4,5 \pm 1,3 \\
4,9 \pm 1,3\end{array}$ & $\begin{array}{l}43, I \pm I_{1}, I \\
32,9 \pm r_{2,3}\end{array}$ \\
\hline
\end{tabular}

(r) Première période : éclairage $7 \mathrm{~h}-2 \mathrm{I} \mathrm{h}$, moyenne des trois dernières semaines.

First period : light from 7 am to 9 pm, average of the last three weeks.

Deuxième période : éclairage $20 \mathrm{~h}-$ Io $\mathrm{h}$, moyenne des trois premières semaines.

Second period : light from $8 \mathrm{pm}$ to $\mathrm{Io}$ am, average of the first threc weeks.

rement permettant le rétablissement d'une consommation nocturne très largement prédominante. Toutefois cette adaptation nécessite un minimum de 4 semaines et ne paraît pas complètement achevée chez certains sujets au bout de 7 semaines.

Malgré ces perturbation: dans la répartition des repas on n'observe pas de modifications du niveau d'ingestion même à court terme.

Si d'un point de vue zootechnique un tel décalage d'horaire paraît donc sans conséquences importantes il n'en est pas de même sur le plan physiologique puisque le rythme d'ingestion des aliments semble bien être un facteur important dans l'établissement d'autres rythmes biologiques notamment celui de la sécrétion des corticostéroïdes surrénaliens ('TAKahashi, Inous et 'TAKAhashi, r977).

Accepté pour publication en octobre 1977.

\section{Summary}

Feeding behaviour of wild rabbits in captivity.

II. - Changes induced by light phase shift.

The feeding pattern of three wild rabbits reared in captivity was recorded. During 4 weeks, the daily light period ranged from 7.00 a.m. to 9.00 p.m. The following 7 weeks it was changed to 8.00 p.m. and ro a.m.

The shift of the light phase did not modify the quantitative feed intake whereas the circadian distribution of feed intake progressively changed within about 4 weeks towards an adaptation to the new light period. In some individuals, this adaptation was not entirely achieved after 7 weeks. 


\section{Références bibliographiques}

Ardisson J. L., Dolisi C., Camous J. P., Gastaud M., i974. Influence de l'éclairement sur le comportement alimentaire du Chien. Arch. Sci. Physiol., 28, I73-I8I.

Cherubin M., r974. Eitude du comportement alimentaire du Lapin domestique du sevrage à la ${ }^{\circ} 8^{\circ}$ semaine. Influence de quelques facteurs extrinsèques sur les paramètres de l'ingestion alimentaire. Thèse Doct. Sp. Montpellier.

LE MAGNFN J., TALLON S., I966. La périodieité spontanée de la prise d'aliments ad libitum du rat blanc. $J$. Physiol., Paris, 58, 328-349.

Prud'ion M., Cari,Es Y., Goussopouros J., KoEmr, P. F., 1972. Enregistrement graphique des consommations d'aliments solide et liquide du lapin domestique nourri ad libitum. $A n n$. Zootech., 21, 45I-460.

Prudion M., Cherubin M., Carl,fis Y., Goussopolios J., 1975. Effets de différents niveaux de restriction hydrique sur l'ingestion d'aliments solides par le Lapin. Ann. Zootech., 24, 299-3 I0.

Prud'hon M., Goussopolios J., 1976. Comportement alimentaire du Lapin de garenne en captivité. Ann. Zootech., 25, 407-4IO.

Sanderson J. D., VAN DER WEELE D. A., 1975. Analysis of feeding patterns in normal and vagotomized rabbits. Physiol. Behav., 15, 357-364.

Takarashi K., Inoue K., Takahashi Y., I977. Parallel shift in circadian rythms of adrenocortical activity and food intake in blinded and intact rats exposed to continuous illumination. Endocrinology, 100, 1097-1107. 